\title{
Space-Based Lidar Systems
}

\author{
Xiaoli Sun \\ NASA Goddard Space Flight Center, Code 694. Greenbelt, Maryland 20771. USA \\ xiuoli.sun-1@nasa.gov
}

\begin{abstract}
An overview of space-based lidar systems is presented, from the first laser altimeter on APOLLO 15 mission in 1971 to the Mercury Laser Altimeter on MESSENGER mission currently in orbit, and those currently under development.

OCIS codes: 280.3640 Lidar; 000,3100 Instruments, apparatus, and components common to the science
\end{abstract}

\section{Introduction}

Lidar, which stands for Light Detection And Ranging, is a powerful tool in remote sensing from space. Compared to radars, lidars operate at a much shorter wavelength with a much narrower beam and much smaller transmitter and receiver. Compared to passive remote sensing instruments, lidars carry their own light sources and can continue measuring day and night, and over polar regions. There are mainly two types of lidars depending on the types of measurements. Lidars that are designed to measure the distance and properties of hard targets are often called laser rangers or laser altimeters. They are used to obtain the surface elevation and global shape of a planet from the laser pulse time-of-flight and the spacecraft orbit position. Lidars that are designed to measure the backscattering and absorption of a volume scatter, such as clouds and aerosol, are often just called lidars and categorized by their measurements, such as cloud and aerosol lidar, wind lidar, $\mathrm{CO}_{2}$ lidar, and so on. The advantages of space-based lidar systems over ground based lidars are the abilities of global coverage and continuous measurements.

\section{Early Space-Based Laser Altimeters}

The first space-based lidar instrument was a laser ranger developed by the United States on the Apollo 15 mission in 1971 using a flash lamp pumped ruby laser at 3.75 pulses per minute. The Apollo 15, 16, and 17 missions combined, made a few thousand measurements of the lunar surface around the equator. With the advent of diode pumped lasers in the late $1980 \mathrm{~s}$, the lifetime, efficiency, resolution and the mass of lasers and space lidar all improved dramatically. The first space-based lidar that used a diode pumped Nd:YAG laser was the Mars Orbiter Laser Altimeter (MOLA) developed at the National Aeronautic and Space Administration (NASA) Goddard Space Flight Center (GSFC) on the Mars Observer mission in 1992. Unfortunately, the spacecraft developed a problem and did not reach the Mars orbit. A smaller laser ranger with similar laser and detector developed by the Naval Research Lab flew on the Clementine mission to the Moon in 1994 and provided a global topographic map of the entire lunar surface at a few hundred-meter precisions. A small laser ranger developed by the Johns Hopkins University Applied Physics Laboratory on board the Near Earth Asteroid Rendezvous (NEAR) mission, launched in 1994, successfully measured the topography and shape of the near Earth asteroid 433 Eros.

A second MOLA was built by NASA GSFC and launched in 1997 on board Mars Global Surveyor (MGS). MOLA was both a laser ranger and a laser radiometer. It measured not only the laser pulse time-of-flight but also the pulse width and energy, which were to correct the range walk caused by the signal amplitude variation, to infer the surface slope from the laser pulse broadening, and to estimate the surface reflectance to the laser light. MOLA onboard science algorithm tracked the ground surface returns and dynamically adjusted the receiver detection threshold and range gate to keep the instrument at the highest receiver sensitivity. MOLA made 650 million Mars surface topographic measurements over one full Martian year (two Earth years) at about 0.25 meter precision. The overall measurement accuracy was limited by the spacecraft orbit position and laser footprint location determination, which was about 1 meter in radial direction and a few hundred meters cross track. The MOLA detector noise, which was a function of the solar background light in the receiver field of view, was also used to produce a passive radiometry map of Mars at $1064 \pm 1 \mathrm{~nm}$ wavelength.

\section{Lidar Experiments on the Space Shuttles}

Two space based lidar systems were flown on the Space Transportation Systems (STS), or Space Shuttles, as technology path finders. Lidar In-space Technology Experiment (LITE) developed by NASA's Langley Research Center (LaRC) was launched in 1994 aboard STS-64. LITE was an atmosphere backscattering lidar using diodepumped Q-switched Nd:YAG laser at 1064, 532, and $355 \mathrm{~nm}$ wavelength and provided valuable data on the backscattering properties of clouds and aerosols as well as surface returns. The Shuttle Laser Altimeter (SLA) developed by NASA GSFC flew on STS-72 in 1996 and STS-85 in 1997 along with several other science payload 
instruments. SLA demonstrated laser surface elevation measurements through Earth's atmosphere and the use of laser altimeter to study vegetation coverage.

\section{Earth Orbiting Lidars}

Two earth orbiting lidars followed the lidar experiments on the Space Shuttle. The first was the Geoscience Laser Altimeter System (GLAS) on the Ice, Cloud and land Elevation Satellite (ICESat) developed at NASA GSFC and launched in January 2003. GLAS had one surface altimetry channel at $1064 \mathrm{~nm}$ wavelength and two cloud and aerosol backscattering profile channels at $1064 \mathrm{~nm}$ and $532 \mathrm{~nm}$, respectively. ICESat completed its science mission in 2009 with about 2 billion laser shot measurements at 2 to $3 \mathrm{~cm}$ range precision. ICESat/GLAS provided unprecedented data set of ice sheets in Antarctica and Greenland, sea ice in the Arctic, land and vegetation in midlatitude, and atmosphere back scattering profiles across the globe.

The Cloud-Aerosol Lidar and Infrared Pathfinder Satellite Observation (CALIPSO) satellite developed by NASA LaRC and Ball Aerospace \& Technology Corporation was launched June 2006. CALIPSO lidar measures the atmosphere backscattering profiles at $532 \mathrm{~nm}$ and $1064 \mathrm{~nm}$ in concert with seven other Earth observing satellites in the "A-Train.".CALIPSO lidar has accumulated more than 2 billion laser pulse measurements and provided new insight into the roles that clouds and atmospheric aerosols play in regulating Earth's climate.

\section{Recent Planetary and Lunar Laser Altimeters}

The Mercury Laser Altimeter (MLA) developed at NASA GSFC on board the MErcury Surface, Space ENvironment, GEochemistry, and Ranging (MESSENGER) missions was launched in August 2004 and entered Mercury's orbit in March 2011. MLA was a miniaturized MOLA but for a much harsher thermal environment. MLA had to make range measurement from a highly elliptical spacecraft orbit around the planet and a laser beam-pointing angle always perpendicular to the sun light due to the spacecraft thermal constraints. As a result, MLA often operates in off nadir direction and over great distances. Despite the harsh thermal environment and unfavorable measurement conditions, MLA has made topographic measurements of Mercury northern hemisphere and continues to operate to this date.

A small lidar developed by NEC and TOSHIBA was on board the Hayabusa mission of Japan, which was launched in 2003, successfully ranged to asteroid Itokawa after orbit insertion in 2005. A small atmosphere backscattering lidar developed in Canada on board the Phoenix Mars Lander was launched in 2007 and successfully measured the Mars atmosphere backscattering profiles until the mission completed in 2008.

Several laser altimeters have flown around the lunar orbit in recent years. The laser altimeter (LALT) developed by NEC Corporation on board the Japanese lunar explorer Selenological and Engineering Explorer (SELENE or Kaguya) was launched in September 2007. LALT made 13 million topographic measurements of the moon at about 5 meter precision and accuracy. The laser altimeter developed by China on board the Chang'E-1 spacecraft launched in October 2007 made about 8 million topographic measurements of the moon. The Lunar Laser Ranging Instrument (LLRI) on board the Chandrayaan spacecraft developed by India was launched in October 2008 and made several million lunar topographic measurements.

The Lunar Orbiter Laser Altimeter (LOLA), developed at NASA GSFC, was launched June 2009 on board the Lunar Reconnaissance Orbiter (LRO) and continues to operate to date. LOLA is the first multi-beam laser altimeter in space. A diffractive optical element (DOE) was mounted on top of the laser beam expander that splits the laser beam into five beams. There are five independent receiver channels, each looking at one laser spot on ground. As a result, it gives not only the surface elevation, but also the slope and directions from a single laser shot. LOLA also provided the surface reflectance measurements and "sees" inside the permanently shadowed craters in polar regions of the moon. To date, LOLA has made about 5 billion lunar topographic measurements.

\section{Space Based Lidar Currently Under Development}

There are several space-based lidar systems currently being built, including the ADM-Aeolus wind lidar for Earth by the European Space Agency (ESA) scheduled to launch in 2013; the Bepi-Colombo Laser Altimeter (BELA) on the Bepi-Colombo mission by ESA to be launched in 2014; and the Advanced Topographic Laser Altimeter System (ATLAS) on the ICESat-2 mission by NASA GSFC to be launched in 2016. Several advanced space based lidar systems are also being planned for near future, including several lidar systems for the International Space Station (ISS), the $\mathrm{CO}_{2}$ lidar for NASA's Active Sensing of $\mathrm{CO}_{2}$ Emission over Nights, Days, and Seasons (ASCENDS) mission, and the laser altimeter for the Jupiter icy moon mission by ESA to be launched later in this decade. 